\title{
RESPOSTA DE GENÓTIPOS DE MILHO AO ESTRESSE SALINO EM CONDIÇÕES HIDROPÔNICAS
}

\author{
Lilia Willadino ${ }^{1 *}$; Marcos Henrique Brainer Martins²; Terezinha Rangel Camara ${ }^{3}$; Arnobio \\ Gonçalves Andrade ${ }^{4}$; Gilberto Dias Alves ${ }^{5}$ \\ ${ }^{1}$ Depto. de Biologia - UFRPE, R. Dom Manoel de Medeiros, s/n - CEP: 52.171-900 - Recife, PE. \\ ${ }^{2}$ Engenheiro Agrônomo - UFRPE. \\ ${ }^{3}$ Depto. de Química - UFRPE. \\ ${ }^{4}$ Depto. de Biologia - UFRPE. \\ ${ }^{5}$ Pós-Graduando do Depto. de Biologia - UFRPE. \\ *e-mail: lilia@truenet.com.br
}

RESUMO: Quatro genótipos de milho (Zea mays L.), Jatinã C3 anão, BR-201, V-190 e LW-1, foram avaliados quanto à tolerância ao estresse salino $(0,50$ e $100 \mathrm{mM} \mathrm{NaCl})$ em condições hidropônicas. As plantas submetidas a $100 \mathrm{mM}$ de $\mathrm{NaCl}$ durante 29 dias apresentaram uma redução na produção de matéria seca superior a $50 \%$, e uma redução na área foliar superior a $75 \%$, quando comparadas ao tratamento controle ( $0 \mathrm{mM} \mathrm{NaCl}$ ). Dentre os quatro genótipos estudados, a linha pura $\mathrm{LW}-1$ apresentou produção de matéria seca e área foliar inferiores aos demais. $O$ aumento dos níveis de sal na solução resultou em uma diminuição da taxa de crescimento da parte aérea e da raiz, de todos os genótipos. 0 sistema radicular apresentou os maiores teores de sódio e também os maiores decréscimos na taxa de crescimento relativo, evidenciando o efeito negativo da acumulação deste cátion. $O$ número e o tamanho dos estômatos não sofreram alterações em conseqüência do estresse salino.

Palavras-chave: salinidade, tolerância, taxa de crescimento, área foliar

\section{RESPONSE OF MAIZE GENOTYPES TO SALINE STRESS IN HYDROPONIC CONDITIONS}

ABSTRACT: Four maize (Zea mays L.) genotypes, Jatinã C3 anão, BR-201, V-190 and LW-1, grown under hidroponic conditions, were evaluated for their tolerance to saline stress imposed by three salt levels $(0,50$ and $100 \mathrm{mM} \mathrm{NaCl})$. Plants submitted to $100 \mathrm{mM} \mathrm{NaCl}$ for 29 days had $50 \%$ lower dry matter production and $75 \%$ reduced leaf area, as compared to the control $(0 \mathrm{mM} \mathrm{NaCl})$ plants. Among the four genotypes studied, LW-1, a pure line, produced the least dry matter and leaf area. The increase salt levels in solution decreased the growth rate of both shoot and root in all genotypes. Roots had the largest sodium concentration and also showed the largest decrease in the relative growth rate, demonstrading the negative effect of sodium accumulation. The number and size of the stomata were not affected by saline stress.

Key words: salinity, tolerance, growth rate, leaf area

\section{INTRODUÇÃO}

A redução do crescimento e da produção das plantas, como conseqüência do estresse salino, vem sendo estudada há vários anos por um grande número de pesquisadores. Estes estudos visam, além de mensurar os efeitos da salinidade sobre a planta, elucidar mecanismos fisiológicos e bioquímicos vinculados à tolerância ou sensibilidade ao estresse. Vários parâmetros são tradicionalmente utilizados nestes estudos, podendo-se destacar: produção de matéria seca (Willadino et al., 1992; Azevedo Neto \& Tabosa, 1998), teores de elementos minerais tais como sódio e potássio (Cramer et al., 1989; Izzo et al., 1993), mecanismos de ajustamento osmótico (Saneoka et al., 1995; Kishor et al., 1995), entre outros. Tais estudos visam elucidar os mecanismos de tolerância, e são de fundamental importância para os trabalhos de seleção de cultivares tolerantes, tanto no melhoramento clássico, como na engenharia genética, para a obtenção de plantas transgênicas tolerantes. A salinização, muitas vezes decorrente do manejo inadequado do solo e da água, pode levar ao abandono de áreas nobres para a agricultura, por torná-las inviáveis à exploração agrícola, devido às drásticas reduções na produtividade das culturas. A utilização de cultivares tolerantes apresenta-se como uma alternativa viável para o reaproveitamento destas áreas marginalizadas. $O$ presente 
trabalho teve como objetivo comparar a linha pura LW-1, obtida através da cultura de anteras (Willadino et al.,1995), com as cultivares Jatinã C3 anão, BR-201 e V-190, quanto a sua tolerância à salinidade em cultivo hidropônico.

\section{MATERIAL E MÉTODOS}

O material vegetal utilizado no experimento constou de quatro genótipos de milho: a cultivar Jatinã C3 anão, utilizada na região Nordeste e considerada relativamente tolerante à salinidade; a cultivar V-190, procedente da Colômbia, onde constitui uma população nativa adaptada às condições de deficiência hídrica; o híbrido duplo BR-201, reconhecido como tolerante à acidez do solo e a linha pura LW-1, obtida pelo grupo, através da cultura de anteras. O experimento foi conduzido em condições de casa-de-vegetação. Inicialmente, foram semeadas 40 sementes de cada genótipo em copos plásticos contendo areia lavada, as quais foram irrigadas diariamente com água destilada. O material foi mantido nestas condições durante 10 dias. Após este período, foram selecionadas 24 plântulas de cada genótipo para o experimento de avaliação de tolerância à salinidade, em condições hidropônicas. As plântulas foram então transferidas para bandejas de plástico $(0,47 \mathrm{x}$ $0,40 \times 0,17 \mathrm{~m}$ ) contendo, cada uma, $14 \mathrm{~L}$ de solução nutritiva completa de Hoagland (Epstein, 1989), acrescida ou não de $\mathrm{NaCl}$ conforme os tratamentos adotados: 0; 50 e $100 \mathrm{mM}$ de $\mathrm{NaCl}$. As plântulas foram fixadas sobre as bandejas em tampas vazadas, tendo suas raízes imersas na solução. Cada uma das 24 bandejas recebeu uma plântula de cada genótipo. As soluções receberam aeração ininterruptamente durante todo o período experimental. O nível de condutividade elétrica da solução nutritiva de cada tratamento foi monitorado a cada dois dias e mantido a aproximadamente: 2,01; 7,78 e 12,38 dS. $\mathrm{m}^{-1}$ para os níveis de 0,50 e $100 \mathrm{mM}$ de $\mathrm{NaCl}$, respectivamente. Aos 14 e 29 dias de tratamento (plantas com 24 e 39 dias de cultivo, respectivamente) o material foi coletado e determinou-se a área foliar total, por meio de um LICOR-3000A, e a quantidade de matéria seca da parte aérea e da raiz. Os teores de sódio e potássio da parte aérea e raiz foram analisados após digestão nitroperclórica do material seco, por espectofotometria de chama (Malavolta, 1989). Para determinação do número e tamanho dos estômatos, foi utilizada uma ocular milimetrada acoplada a fotomicroscópio com objetiva de 40X. A taxa de crescimento relativo (TCR) foi calculada para a parte aérea, raiz e total (parte aérea+raiz), pela equação: $T C R=L n P_{2}-L n P_{1} / t_{2}-t_{1}$, onde: $P_{2}$ e $P_{1}$ são o peso da matéria seca no tempo $2\left(t_{2}=29\right.$ dias) e no tempo 1 ( $t_{1}=14$ dias) (Alves et al., 1995).

$O$ desenho experimental utilizado foi um fatorial $4 \times 3 \times 2$ (genótipos $x$ níveis de $\mathrm{NaCl} \times$ tempo), com quatro repetições por tratamento. $\mathrm{O}$ efeito dos tratamentos foi avaliado estatisticamente através da análise de variância e aplicouse 0 teste de Tukey a $5 \%$ de probabilidade para a comparação de médias.

\section{RESULTADOS E DISCUSSÃO}

O aumento do nível de sal na solução resultou numa redução do crescimento dos quatro genótipos estudados. As plantas submetidas a $100 \mathrm{mM}$ de $\mathrm{NaCl}$ apresentaram um decréscimo na área foliar e na produção de matéria seca superiores a $50 \%$, quando comparadas ao controle (TABELA 1). Resultados similares foram observados por Saneoka et al. (1995), ao submeterem linhagens de milho ao estresse salino utilizando soluções crescentes de $\mathrm{NaCl}$ : $42 \mathrm{mM}$ durante 6 dias, $85 \mathrm{mM}$ durante 15

TABELA 1 - Área foliar (AF) e produção de matéria seca da parte aérea (MSPA), da raiz (MSR) e total (MST). Média de quatro genótipos de milho submetidos, durante 14 e 29 dias, a diferentes níveis de $\mathrm{NaCl}$.

\begin{tabular}{ccccccccc}
\hline $\mathrm{NaCl} m M$ & MSPA $(\mathrm{g})$ & $\mathrm{MSR}(\mathrm{g})$ & $\mathrm{MST}(\mathrm{g})$ & $\mathrm{AF}\left(\mathrm{cm}^{2}\right)$ & $\mathrm{MSPA}(\mathrm{g})$ & $\mathrm{MSR}(\mathrm{g})$ & $\mathrm{MST}(\mathrm{g})$ & $\mathrm{AF}\left(\mathrm{cm}^{2}\right)$ \\
\hline & - & & & & & & & \\
\hline 0,0 & $7,40 \mathrm{a}$ & $1,87 \mathrm{a}$ & $9,27 \mathrm{a}$ & $1344 \mathrm{a}$ & $40,23 \mathrm{a}$ & $12,79 \mathrm{a}$ & $53,12 \mathrm{a}$ & $4224 \mathrm{a}$ \\
50,0 & $4,94 \mathrm{~b}$ & $1,71 \mathrm{a}$ & $6,65 \mathrm{~b}$ & $926 \mathrm{~b}$ & $26,41 \mathrm{~b}$ & $7,97 \mathrm{~b}$ & $34,38 \mathrm{~b}$ & $2959 \mathrm{~b}$ \\
100,0 & $2,41 \mathrm{c}$ & $0,99 \mathrm{~b}$ & $3,40 \mathrm{c}$ & $499 \mathrm{c}$ & $11,03 \mathrm{c}$ & $3,01 \mathrm{c}$ & $14,05 \mathrm{c}$ & $1072 \mathrm{c}$ \\
\hline
\end{tabular}

Médias numa mesma coluna seguidas de uma mesma letra não apresentam diferença significativa a nível de $5 \%$ de probabilidade pelo Teste de Tukey. 
dias e $127 \mathrm{mM}$ durante 7 dias, num total de 28 dias de estresse. Izzo et al. (1993), demostraram que a redução do crescimento de plantas de milho em condições de estresse salino está associada a uma redução do potencial osmótico da planta, e não ao potencial de pressão, o que indica um ajustamento osmótico decorrente da síntese de solutos compatíveis.

A presença do cloreto de sódio na solução nutritiva resultou em um aumento dos teores de sódio na planta (TABELA 2). Na raiz foram detectados os maiores teores deste cátion, indicando um acúmulo do mesmo em detrimento da sua translocação para a parte aérea. A raiz, além de apresentar maiores teores de sódio apresentou também, a menor taxa de crescimento relativo (Figura 1) o que reflete o efeito deletério da acumulação de sódio no tecido.

Paralelamente aos aumentos dos níveis de sódio, observou-se uma diminuição dos teores de potássio na planta como consequência do aumento dos níveis de $\mathrm{NaCl}$ na solução (TABELA 2). Segundo Cramer et al. (1985 e 1989), esta diminuição dos teores de potássio ocorre devido a um aumento da permeabilidade das membranas que é função do deslocamento do cálcio pelo sódio no plasmalema, característico de situações onde a relação $\mathrm{Na}^{+} / \mathrm{Ca}^{2+}$ é elevada.

Dentre os quatro genótipos estudados, o LW-1 apresentou-se como o menos tolerante, quando considerada a produção de matéria seca. (TABELA 3 e 4), área foliar (TABELA 3) e

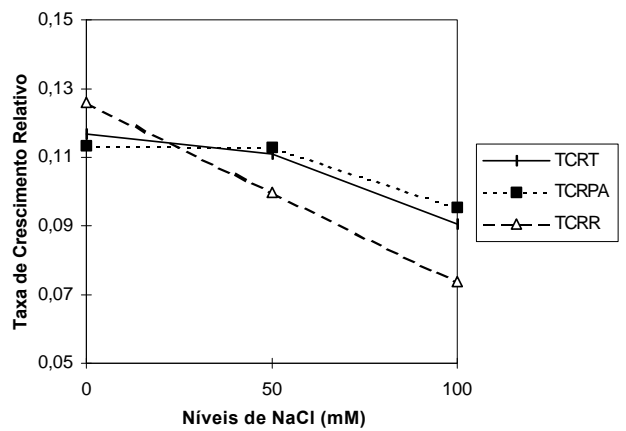

Figura 1 - Taxa de Crescimento Relativo Total (TCRT), da parte aérea (TCRPA) e da raiz (TCRR), de quatro genótipos de milho submetidos a diferentes níveis de $\mathrm{NaCl}$.

TABELA 2 - Teores de sódio e potássio (\%) na parte aérea e raiz. Média de quatro genótipos de milho submetidos, durante 14 e 29 dias, a diferentes níveis de $\mathrm{NaCl}$.

\begin{tabular}{|c|c|c|c|c|c|c|c|c|}
\hline \multirow[b]{3}{*}{$\mathrm{NaCl} \mathrm{mM}$} & \multirow{2}{*}{\multicolumn{4}{|c|}{-- Parte aérea -- }} & \multirow{2}{*}{\multicolumn{4}{|c|}{--- Parte aérea --- $\quad$---- Raiz ---- }} \\
\hline & & & & & & & & \\
\hline & 14 dias & 29 dias & 14 dias & 29 dias & 14 dias & 29 dias & 14 dias & 29 dias \\
\hline 0,0 & $0,06 \mathrm{c}$ & $0,04 \mathrm{c}$ & $0,28 \mathrm{c}$ & $0,26 \mathrm{~b}$ & $7,46 \mathrm{a}$ & $4,13 a b$ & $6,22 \mathrm{a}$ & $2,68 \mathrm{a}$ \\
\hline 50,0 & $0,88 \mathrm{~b}$ & $1,61 \mathrm{~b}$ & $3,17 \mathrm{~b}$ & $5,08 \mathrm{a}$ & $5,24 \mathrm{~b}$ & $4,28 \mathrm{a}$ & $2,68 \mathrm{~b}$ & $1,97 \mathrm{~b}$ \\
\hline 100,0 & $2,37 \mathrm{a}$ & 3,73 a & $3,65 \mathrm{a}$ & $5,58 \mathrm{a}$ & $3,66 \mathrm{c}$ & $3,69 \mathrm{~b}$ & $1,79 \mathrm{c}$ & $1,47 \mathrm{c}$ \\
\hline
\end{tabular}

Médias numa mesma coluna seguidas de uma mesma letra não apresentam diferença significativa a nível de $5 \%$ de probabilidade pelo Teste de Tukey.

TABELA 3 - Produção de matéria seca de parte aérea (MSPA), raiz (MSR), total (MST) e área foliar (AF) dos genótipos Jatinã C3 anão, V-190, BR-201 e LW-1, submetidos, durante 14 e 29 dias, a diferentes níveis de $\mathrm{NaCl}$.

\begin{tabular}{|c|c|c|c|c|c|c|c|c|}
\hline \multirow[t]{2}{*}{ Cultivar } & MSPA (g) & $\operatorname{MSR}(\mathrm{g})$ & MST (g) & $\mathrm{AF}\left(\mathrm{cm}^{2}\right)$ & MSPA (g) & MSR (g) & MST $(g)$ & $\mathrm{AF}\left(\mathrm{cm}^{2}\right)$ \\
\hline & \multicolumn{4}{|c|}{ - - } & \multicolumn{4}{|c|}{ - } \\
\hline Jatinã & $6,27 \mathrm{a}$ & 1,99 a & 8,26 a & $1141 \mathrm{a}$ & 29,21 a & $9,02 \mathrm{a}$ & $38,23 \mathrm{a}$ & 3308 a \\
\hline$V-190$ & $6,09 \mathrm{a}$ & $1,93 \mathrm{a}$ & $8,02 \mathrm{a}$ & $1140 \mathrm{a}$ & 29,24 a & $9,82 \mathrm{a}$ & $39,05 \mathrm{a}$ & 2987 a \\
\hline BR-201 & $4,79 \mathrm{~b}$ & $1,49 \mathrm{~b}$ & $6,28 \mathrm{~b}$ & 953 a & $30,31 \mathrm{a}$ & 9,77 a & 40,08 a & $3252 \mathrm{a}$ \\
\hline LW-1 & $2,52 \mathrm{c}$ & $0,68 \mathrm{c}$ & $3,20 \mathrm{c}$ & $457 \mathrm{~b}$ & $14,93 \mathrm{~b}$ & $3,10 \mathrm{~b}$ & $18,03 \mathrm{~b}$ & $1459 \mathrm{~b}$ \\
\hline
\end{tabular}

Médias numa mesma coluna seguidas de uma mesma letra não apresentam diferença significativa a nível de $5 \%$ de probabilidade pelo Teste de Tukey. 
crescimento relativo (TABELA 5). Nesta linhagem, a produção de matéria seca do sistema radicular sofreu uma redução superior a $50 \%$ no nível de $50 \mathrm{mM}$ de $\mathrm{NaCl}$, enquanto as demais cultivares só apresentaram redução nesta ordem no nível de $100 \mathrm{mM}$ de $\mathrm{NaCl}$ (TABELA 4). Quando considerada a produção de matéria seca total (parte aérea + raiz), fica evidente um menor potencial produtivo da linha pura LW-1 em todos os tratamentos salinos (TABELA 3). Trabalhos anteriores, realizados pela equipe (Willadino et al., 1995), mostraram que a linha pura LW-1 apresentou uma elevada tolerância à salinidade (137 mM) na fase de germinação, sendo esta tolerância mantida durante os estádios iniciais da plântula. Entretanto, observa-se no presente trabalho que esta tolerância não se expressa em estádios posteriores, ou quando é aumentada a duração do estresse. Esta constatação corrobora a afirmação de outros autores de que a avaliação da tolerância à salinidade em cultivares de milho, não pode se basear em uma única fase do ciclo fenológico (Maas et al., 1983; Willadino et al., 1992).

TABELA 4 - Produção de matéria seca $(\mathrm{g})$ de raiz dos genótipos Jatinã C3 anão, V190, BR-201 e LW-1, submetidos, durante 29 dias, a 0,50 e $100 \mathrm{mM}$ $\mathrm{NaCl}$.

\begin{tabular}{ccccc}
\hline $\mathrm{NaCl}(\mathrm{mM})$ & Jatinã & V-190 & BR-201 & LW-1 \\
\hline 0,0 & $14,25 \mathrm{Aa}$ & $16,82 \mathrm{Aa}$ & $14,56 \mathrm{Aa}$ & $5,55 \mathrm{Ba}$ \\
50,0 & $9,05 \mathrm{Ab}$ & $9,42 \mathrm{Ab}$ & $10,72 \mathrm{Ab}$ & $2,69 \mathrm{Bab}$ \\
100,0 & $3,76 \mathrm{Ac}$ & $3,21 \mathrm{Ac}$ & $4,02 \mathrm{Ac}$ & $1,06 \mathrm{Ab}$ \\
\hline
\end{tabular}

Médias seguidas da mesma letra minúscula, numa mesma coluna, e maiúsculas, numa mesma linha, não apresentam diferença significativa a nível de $5 \%$ de probabilidade pelo Teste de Tukey.

TABELA 5 - Taxa de crescimento relativo ( dia $^{-1}$ ) da parte aérea de quatro genótipos de milho submetidos, durante 14 dias, a diferentes níveis deNaCl.

\begin{tabular}{lrrrr}
\hline $\mathrm{NaCl}(\mathrm{mM})$ & Jatinã & $\mathrm{V}-190$ & $\mathrm{BR}-201$ & $\mathrm{LW}-1$ \\
\hline 0,0 & $0,11 \mathrm{Aa}$ & $0,09 \mathrm{Aa}$ & $0,12 \mathrm{Aa}$ & $0,11 \mathrm{Aa}$ \\
50,0 & $0,09 \mathrm{Aa}$ & $0,11 \mathrm{Aa}$ & $0,11 \mathrm{Aa}$ & $0,12 \mathrm{Aa}$ \\
100,0 & $0,08 \mathrm{AB}$ a & $0,10 \mathrm{AB}$ a & $0,12 \mathrm{Aa}$ & $0,07 \mathrm{Bb}$ \\
\hline
\end{tabular}

Médias seguidas da mesma letra minúscula, numa mesma coluna, e maiúsculas, numa mesma linha, não apresentam diferença significativa a nível de $5 \%$ de probabilidade pelo Teste de Tukey.
A salinidade não promoveu alteração no número, nem no tamanho (comprimento e largura) dos estômatos. Resultados contrastantes foram observados em outros cultivares de milho, onde detectou-se redução na frequência estomática, como conseqüencia do estresse salino (Radi et al.,1989) e hídrico (Singh \& Singh, 1989). As cultivares V-190 e Jatinã C3 anão apresentaram um maior número de estômatos. $\mathrm{mm}^{-2}$ (TABELA 6). O híbrido BR-201, apresentou estômatos com maior comprimento, quando comparado a cultivar Jatinã C3 anão, o que indica que tanto a freqüência estomática quanto o tamanho dos estômatos apresentaramse como características intrínsecas de cada genótipo e não como resposta ao estresse salino.

TABELA 6 - Frequência (número. $\mathrm{mm}^{-2}$ ), largura $(\mu \mathrm{m})$ e comprimento $(\mu \mathrm{m})$ dos estômatos de quatro genótipos de milho submetidos, durante 29 dias, a diferentes níveis de $\mathrm{NaCl}$.

\begin{tabular}{lccc}
\hline Cultivar & $\begin{array}{c}\text { Número } \\
\left(\mathrm{mm}^{2}\right)\end{array}$ & $\begin{array}{c}\text { Comprimento } \\
(\mu \mathrm{m})\end{array}$ & $\begin{array}{c}\text { Largura } \\
(\mu \mathrm{m})\end{array}$ \\
\hline Jatinã & $89,31 \mathrm{a}$ & $47,10 \mathrm{~b}$ & $39,15 \mathrm{a}$ \\
V-190 & $89,15 \mathrm{a}$ & $49,02 \mathrm{ab}$ & $37,82 \mathrm{a}$ \\
BR-201 & $76,22 \mathrm{~b}$ & $51,44 \mathrm{a}$ & $43,27 \mathrm{a}$ \\
LW-1 & $79,84 \mathrm{~b}$ & $49,87 \mathrm{ab}$ & $38,98 \mathrm{a}$ \\
\hline
\end{tabular}

Médias seguidas da mesma letra, numa mesma coluna, não apresentam diferença significativa a nível de $5 \%$ de probabilidade pelo Teste de Tukey.

\section{REFERÊNCIAS BIBLIOGRÁFICAS}

AZEVEDO NETO, A.D.; TABOSA, J.N. Avaliação de tolerância à salinidade em cultivares de milho na fase de plântula. In: CONGRESSO NACIONAL DE MILHO E SORGO, 22., Recife,1998. Resumos. Recife:Associação Brasileira de Milho e Sorgo, 1998. 272p.

ALVES, V.M.C.; NOVAES, F.R.; OLIVEIRA, M.F.G.; MOSQUIM, P.R. Açúcares solúveis em quatro híbridos de milho sob omissão e ressuprimento de fósforo. Revista Brasileira de Fisiologia Vegetal, v.7, p.97-105, 1995.

CRAMER, G.R.; EPSTEIN, E.; LAUCHLI, A. Na-Ca interactions in barley seedlings: relastionship to ion transport and growth. Plant Cell Environment, v.81, p.792-797, 1989.

CRAMER, G.R.; LAUCHLI, A.; POLITO, U.S. The displacement of $\mathrm{Ca}^{2+}$ by $\mathrm{Na}^{+}$from the plasmalemma of root cells: a primary response to salt stress? Plant Physiology, v.97, p.207-211, 1985. 
EPSTEIN, E. Mineral nutrition of plants: principles and perspectives. New York: John Willey \& Sons, 1989. 152p.

IZZO, R.; SCAGNOZZI, A.; BELLIGNO, A.; NAVARIIZZO, F. Influence of $\mathrm{NaCl}$ treatment on $\mathrm{Ca}, \mathrm{K}$ and $\mathrm{Na}$ interrelations in maize shoots. In: FRAGOSO,M.A.C.; BEUSICHEM, M.L. (Ed.) Optimization of plant nutrition. Netherlands: Kluwer Academic Publishers, 1993. p.577-582.

KISHOR, P.B.K.; HONG, Z.; MIAO, G.H.; HU, C.A.A.; VERMA, D.P.S. Overexpression of pyrroline-5carboxilate synthetase increases proline production and confers osmotolerance in transgenic plants. Plant Physiology, v.108, p.1387-1394, 1995.

MALAVOLTA, E.; VITTI, G.C.; OLIVEIRA, S.A. Avaliação do estado nutricional das plantas. Piracicaba: Associação Brasileira para Pesquisa da Potassa e Fosfato, 1989. 97p.

MAAS, E.V.; HOFFMAN, G.J.; CHABA, G.D.; SHANNON, M.C. Salt sensitivity of corn at various stages. Irrigation Science, v.4, p.45-57, 1983.

RADI, A.F.; HEIKAL, M.M.; ABDEL-RAHAMAN, A.M.; EL-DEEP, B.A.A. Interactive effects of salinity and phytormones on growth and plants water relationship parameters in maize and sanflower plants. Acta Agronomica Hungarica, v.38, p.271-282, 1989.
SANEOKA, H.; NAGASKA, C.; HAHN, D.T.; YANG, W.J.; PREMACHANDRA, G.S.; JOLY, R.J.; RODHES, D. Salt tolerance of glycinebetainedeficient and containing maize lines. Plant Physiology, v.107, p.631-638, 1995.

SINGH, B.R.; SINGH, D.P. Effects of irrigation on stomatal pattern of sorghum, maize and pearl millet. Crop Research, v.2, p.54-58, 1989.

WILLADINO, L.; CAMARA, T.R.; ANDRADE, A.G.; TABOSA, J.N. Tolerancia de cultivares de maiz a la salinidad en diferentes fases de desarrollo. In: SIMPOSIO NACIONAL SOBRE NUTRICIÓN MINERAL DE LAS PLANTAS, 4., Alicante, 1992. Anais. Alicant: Universidad de Alicante, 1992. p.487-494.

WILLADINO, L.; CAMARA, T.R.; SANTOS, M.A.; TORNE, J.M. Obtenção de uma linhagem de milho tolerante ao estresse salino mediante a cultura de anteras. Pesquisa Agropecuária Brasileira, v.30, p.1313-1318, 1995.

Recebido para publicação em 10.03.99 Aceito para publicação em 03.08.99 